\title{
Following the Kinetics of Barium Titanate Nanocrystal Formation in Benzyl Alcohol Under Near-Ambient Conditions
}

\author{
Sjoerd A. Veldhuis, * Tomasz M. Stawski, Pablo Gonzalez Rodriguez, Huiyu Yuan, \\ Rogier Besselink, Liane G. Benning, and Johan E. ten Elshof*
}

In complex chemical syntheses (e.g., coprecipitation reactions), nucleation, growth, and coarsening often occur concurrently, obscuring the individual processes. Improved knowledge of these processes will help to better understand and optimize the reaction protocol. Here, a form-free and model independent approach, based on a combination of time-resolved small/ wide-angle X-ray scattering, is employed to elucidate the effect of reaction parameters (such as precursor concentration, reactant stoichiometry, and temperature) on the nucleation, crystallization, and growth phenomena during the formation of nanocrystalline barium titanate. The strength of this approach is that it relies solely on the total scattered intensity (i.e., scattering invariant) of the investigated system, and no prior knowledge is required. As such, it can be widely applied to other synthesis protocols and material's systems. Through the scattering invariant, it is found that the amorphous-to-crystalline transformation of barium titanate is predominantly determined by the total amount of water released from the gel-like barium hydroxide octahydrate precursor, and three rate-limiting regimes are established. As a result of this improved understanding of the effect of varying reaction conditions, elementary boundary conditions can be set up for a better control of the barium titanate nanocrystal synthesis.

\section{Introduction}

Nanocrystals (NCs) often display very different optoelectronic, chemical, or magnetic properties than their bulk counterparts, mainly imparted by their strong size-dependent characteristics. Over the last decades, great progress has been made to fabricate and manipulate materials at the nanoscale, which assisted the trend of device miniaturization. ${ }^{[1]}$ As devices and device components are fabricated at ever smaller sizes, a fundamental understanding of the phase formation is a prerequisite to exert

Dr. S. A. Veldhuis, ${ }^{[+]}$Dr. P. Gonzalez Rodriguez, Dr. H. Yuan,

Prof. J. E. ten Elshof

Inorganic Materials Science Group

MESA+ Institute for Nanotechnology

University of Twente

P. O. Box 217, 7500 AE Enschede, The Netherlands

E-mail: s.a.veldhuis@gmail.com, sveldhuis@ntu.edu.sg;

j.e.tenelshof@utwente.nl

The ORCID identification number(s) for the author(s) of this article can be found under https://doi.org/10.1002/smll.201802003.

[+]Present address: Energy Research Institute at NTU (ERI@N), Research Techno Plaza, X-Frontier Block Level 5, 50 Nanyang Drive, Singapore 637553 microstructural and morphological control (e.g., monodispersity) at these length scales. Due to the high surface-to-volume ratio of $\mathrm{NCs}$, however, it becomes challenging to discern between the nucleation and growth processes. In order to form monodisperse nanocrystals, typically a single and unique nucleation event (i.e., burst nucleation) is required to prevent further nucleation steps as they interfere with the growth of the as-formed nuclei. As nucleation presents a large energy barrier, high levels of supersaturation facilitate overcoming this barrier. For example, the hot-injection technique is able to overcome the nucleation barrier through the swift injection of precursors into a ligand-containing solution at high temperature. ${ }^{[2]}$ This method has been successfully employed to synthesize a wide range of metal and metal chalcogenide quantum dots, ${ }^{[3]}$ and more recently, lead halide perovskite nanocrystals. ${ }^{[4]}$ The short reactions times (i.e., time scale in seconds), however, pose a challenge to discern the subsequent reaction processes. Methods based on premixing the precursors at low temperatures, while initiating their crystallization at elevated temperatures, provide a more suitable approach to follow the nucleation and growth phenomena, due to extended reaction times. This method has been widely adopted in the nonaqueous sol-gel synthesis of monodisperse binary and ternary metal oxide $\mathrm{NCs},{ }^{[5]}$ whereby the oxygen is typically provided by either the solvent or the metal alkoxide/acetylacetonate precursors. ${ }^{[6]}$ Hydrolytic sol-gel routes, on the other hand, involve the hydrolysis

DOI: $10.1002 / \mathrm{smll} .201802003$ 
and subsequent condensation of metal alkoxide precursors (in aqueous basic or acidic conditions). ${ }^{[7]}$ These water-based reactions are often limited by (1) the high reactivity of water toward the metal alkoxide precursors, (2) the immiscibility of water with the organic solvents in which the reaction takes place (i.e., inhomogeneous distribution), or (3) reaction temperatures above the boiling point of water. ${ }^{[1]}$ Hence, judicious control over reaction parameters is required to form high quality NCs. Previously, we proposed a facile one-pot alkoxide-hydroxide precipitation of nanocrystalline barium titanate $\left(\mathrm{BaTiO}_{3}\right.$; BTO) powders in benzyl alcohol under near ambient reaction conditions. ${ }^{[8]}$ We showed that, the Ti-alkoxide precursor was hydrolyzed by water, slowly released from the $\mathrm{Ba}(\mathrm{OH})_{2} \cdot 8 \mathrm{H}_{2} \mathrm{O}$ precursor under mild heating, and subsequently condensed into $\approx 10 \mathrm{~nm}$ sized BTO NCs. Despite our understanding of the final product at predetermined reaction times and temperatures, we do not yet know which processes occur at the earlier stages of BTO formation, or how these processes proceed. The coprecipitation reaction involves concomitant nucleation, growth and coarsening processes, and the individual reaction steps are frequently obscured. ${ }^{[9]}$ To overcome this, we followed the amorphous-to-crystalline phase transition of BTO using a combination of time-resolved small/ wide angle X-ray scattering (SAXS/WAXS) ${ }^{[10]}$ and X-ray diffraction (XRD). In this report, we combined the above methods with high-resolution microscopy of the formed phases to elucidate the effect that varying reaction conditions (i.e., reagent concentrations, temperature, water content, and precursor stoichiometry) have on the reaction rate and mechanism of BTO nanocrystal formation. Using form-free and/or model independent analysis of SAXS curves we quantified the effect of reaction conditions on the nucleation, crystallization, and growth phenomena during the synthesis. We could show that the rate and extent of crystalline phase formation was predominantly determined by the amount of water present and that for nonstoichiometric precursor ratios a more complex relationship between reaction parameters existed.

\section{Model Independent SAXS Data Analysis}

A sample, which is either particulate or bicontinuous (nonparticulate) in nature and at the same time exhibits nanometer-range electronic density variations, will scatter photons from the monochromatic collimated X-ray beam that passes through said sample. In our case, these density fluctuations may arise from a homogeneous suspension of BTO particles (with electron density $\rho$ ) in a solvent matrix of different electron density, $\rho_{0}$ (or similarly from porosity within a particle). The recorded scattering intensity, $I(q)$, is proportional to the square of electron density difference, $(\Delta \rho)^{2}$, between the BTO particles and the benzyl alcohol solvent matrix. Conventionally, $I(q)$ is plotted versus the magnitude of the scattering vector, $q$, and is related to the scattering angle $(2 \theta)$ and the wavelength $(\lambda)$ of the incident beam via

$q=\frac{4 \pi}{\lambda} \sin \theta$
For particulate systems, as we describe here, the scattered intensity can be expressed as a product of the number density of particles, $N$, the scattering contrast, $(\Delta \rho)^{2}$, particle volume, $V$, and a single particle form factor $P(q)$

$I(q)=N \cdot(\Delta \rho)^{2} \cdot V^{2} \cdot P(q)$

One can also define $I_{0}=N \cdot(\Delta \rho)^{2} \cdot V^{2}$ as a convenient parameter to scale the form factor against the scattered intensity, since $P(q \rightarrow 0)=1$. Typically, form factors express the shape of individual scatterers and the appropriate $P(q)$ functions are derived for a variety of simple geometries. ${ }^{[11]}$ Furthermore, more complicate assemblies of particles are often referred to as form factors, like, e.g., mass-fractal aggregates, ${ }^{[12]}$ or polymer coils, ${ }^{[11 \mathrm{~b}]}$ as long as the individual assemblies are noninteracting and monodisperse in nature. In this study, we treat the aggregate of primary particles as an equivalent collection of polydisperse particles. This is a common approach used for cases when the shape of particles can be approximated by spheres of radius, $R$. In such cases, the form factor $P(q)$ product is expressed as:

$P(q, R)=\left(3 \frac{\sin (q R)-q R \cdot \cos (q R)}{(q R)^{3}}\right)^{2}$

For polydisperse spherical particles, however, Equation (3) is no longer valid, and the size distribution function $D(r)$ has to be included in the expression for the form factor. The averaged form factor of spheres is then given by Equation (4)

$\langle P(q, \bar{R}, \sigma)\rangle=\frac{\int_{0}^{\infty} D(q, \bar{R}, \sigma) P(q, r) r^{3 d} d r}{\int_{0}^{\infty} D(q, \bar{R}, \sigma) r^{3 d} d r}$

where $\bar{R}$ denotes the averaged radius, and $\sigma$ is the variance of the distribution. The parameter $d$ takes either values of 0,1 , or 2. Depending on the value of $d$, the average radius yields the intensity, volume (mass), or number weighted value of $\bar{R}$, respectively. In such case, $I_{0}$ is described as $I_{0}=N \cdot(\Delta \rho)^{2} \cdot\left\langle V^{2}\right\rangle$, where $\left\langle V^{2}\right\rangle$ denotes the distribution-averaged squared volume of a spherical particle, because $\langle P(q \rightarrow 0)\rangle=1$.

In order to characterize a system of polydisperse spherical particles, typically, a certain form of $D(r)$ is assumed and the values of $\bar{R}$ and $\sigma$ are obtained from fitting the expression to the intensity data. The choice of a particular $D(r)$ function is usually limited to only a small number of mathematical expressions, which may potentially describe a given physical phenomenon. In quickly evolving and highly polydisperse systems, a simple form of $D(r)$ is often not expected, and multimodal distributions have to be considered. A form-free distribution, as discussed below, would then be far more useful for that kind of particulate systems. This can be achieved by, e.g., the structure interference methods and their Monte Carlo derivatives. ${ }^{[13]}$ Pauw et al. have recently released a software package that allows for the finding of form-free size distribution histograms. ${ }^{[14]}$ 


\subsection{Scattering Invariant}

Most of the scattering functions are based on assumptions regarding the particle shape (i.e., $P(q)$ ). The scattering invariant (or Porod invariant) is only dependent on the total scattering volume, and thus remains constant for a given concentration. ${ }^{[15]}$ Consequently, this allows us to monitor the extent of the total particle volume evolution during synthesis. The scattering invariant, $Q_{\text {inv }}$, is determined by

$Q_{\text {inv }}=\int_{0}^{\infty} q^{2} \cdot I(q) \mathrm{d} q$

The high intensity measured at low q, caused by scattering of the largest particles (i.e., large volume contribution), is thus normalized by multiplying with $q^{2}$. Additionally, the average correlation length $\langle L\rangle$, correlation surface $\langle A\rangle$, and correlation volume (or Porod volume) $V_{\mathrm{p}}$, of our investigated system are determined using the scattering invariant ${ }^{[15,16]}$

$\langle L\rangle=\frac{\pi}{Q_{\mathrm{inv}}} \int_{0}^{\infty} q \cdot I(q) \mathrm{d} q=\frac{3}{2} \cdot \frac{\left\langle R^{4}\right\rangle}{\left\langle R^{3}\right\rangle}$

$\langle A\rangle=\frac{2 \pi}{Q_{\text {inv }}} \int_{0}^{\infty} I(q) \mathrm{d} q=\frac{4 \pi}{5} \cdot \frac{\left\langle R^{5}\right\rangle}{\left\langle R^{3}\right\rangle}$

$V_{\mathrm{p}}=\frac{2 \pi^{2}}{Q_{\text {inv }}} \cdot I_{0}=\frac{4 \pi}{3} \cdot \frac{\left\langle R^{6}\right\rangle}{\left\langle R^{3}\right\rangle}$

where $R$ represents a measure of the size of a given phase, the brackets \langle\rangle represent an average, and $I_{0}$ the extrapolated intensity for $q \rightarrow 0: I_{0}=N \cdot(\Delta \rho)^{2} \cdot\left\langle V^{2}\right\rangle$. Consequently, $\langle L\rangle,\langle A\rangle$, and $V_{p}$, represent the volume-weighted average of the typical diameter, cross-section area, and volume of the phases that are present in the dispersion, respectively. In theory, these length-scales are an average of sizes of both the high density phases (particles) and low-density phase (solution in between particles). However, for the dispersions with low particle number densities, interparticle correlations are predominant at larger distances (i.e., outside the measured q-regime), and thus can be neglected. Consequently, these measures represent sizes of the particles in dispersion. In such case, the correlation length describes the average length of all possible lines that can be drawn though a particle in all possible orientations, ${ }^{[15]}$ which is proportional to, but smaller than the particle diameter, since the majority of lines do not go through the particle's center. Similarly, $\langle A\rangle$ represents a volume averaged particle intersection area for all possible cross-sections through a particle.

A representation of the number density of the emerging phase (with changing electron density contrast) is obtained by replacing $I_{0}$ by $N \cdot(\Delta \rho)^{2} \cdot\left\langle V^{2}\right\rangle$ in Equation (8), resulting in $Q_{\text {inv }}=2 \pi^{2} \cdot N \cdot(\Delta \rho)^{2} \cdot\langle V\rangle$. Subsequently, by assuming that $\langle V\rangle^{2} \approx\left\langle V^{2}\right\rangle$, the volume term can be eliminated by the following ratio

$N \cdot(\Delta \rho)^{2}=\frac{1}{4 \pi} \cdot \frac{Q_{\text {inv }}^{2}}{I_{0}}=\frac{1}{4 \pi^{2}} \frac{(2 \pi)^{2} \cdot N^{2} \cdot(\Delta \rho)^{4} \cdot\langle V\rangle^{2}}{N \cdot(\Delta \rho)^{2} \cdot\left\langle V^{2}\right\rangle}$
Moreover, the specific surface area could theoretically be determined by applying Porod's law, where the limit of $q \rightarrow \infty$ for $I(q)=q^{-4}$ is proportional to the specific surface area. However, the intensity is not always proportional to $q^{-4}$, for example, due to surface roughness. In such a case we will find an increasing surface area for decreasing length scales, which becomes infinite for $q \rightarrow \infty{ }^{[17]}$ Nevertheless, the specific surface cannot be infinite since these particles have a minimum size $r_{0} \cdot{ }^{[18]}$ Since this type of extrapolation is not reliable for particles of unknown shapes, we applied a different approach: the correlation surface is proportional to the particle crosssection, which for (slightly) ellipsoidal objects is proportional to its outer surface area. Consequently, the ratio of the correlation surface over its particle volume provides is proportional to its specific surface area

$\frac{\langle A\rangle}{V_{\mathrm{p}}}=\frac{3}{5} \cdot \frac{\left\langle R^{5}\right\rangle}{\left\langle R^{6}\right\rangle} \approx \frac{3}{5} \cdot \frac{\left\langle R^{2}\right\rangle}{\left\langle R^{3}\right\rangle}=\frac{1}{5} \cdot \frac{\mathrm{S}}{\mathrm{V}}$

Although the calculation of the invariant is straightforward, in practice it is difficult to measure from $q \rightarrow 0$ to $q \rightarrow \infty$. If we consider the measured $q$-range as $q_{\mathrm{L}}<q<q_{\mathrm{H}}$, the contributions at $q_{\mathrm{L}} \rightarrow 0$ and $q_{\mathrm{H}} \rightarrow \infty$ can be extrapolated using the Guinier approximation ${ }^{[11 a]}$ and Porod's law, ${ }^{[15]}$ respectively. In this manner, good estimates of $Q_{\text {inv }}$ can be determined.

\section{Results and Discussion}

\subsection{Barium Titanate Formation}

The BTO nanocrystals were formed at $78{ }^{\circ} \mathrm{C}$ after sequential hydrolysis and condensation of titanium (IV) isopropoxide and $\mathrm{Ba}(\mathrm{OH})_{2} \cdot 8 \mathrm{H}_{2} \mathrm{O}$ in benzyl alcohol. The water molecules necessary to initiate the hydrolysis, originated from the Ba hydrate salt, and were released under heating. The extent of crystalline BTO phase formation was followed by measuring the increase of the integrated $(110)$ peak area at $2 \theta \approx 31.3^{\circ}$ in the XRD patterns (Figure 1a). From the onset of crystallization at $-20 \mathrm{~min}$ reaction time (determined from time-resolved X-ray diffraction), both the bulk crystalline volume and crystallite sizes increased rapidly, as observed from the increased intensity and narrowing of the diffraction peak at extended reaction times, respectively. This growth process continued for $\approx 240 \mathrm{~min}$, after which a plateau was reached (i.e., no further crystallite growth). In order to monitor the changes during the amorphous phase and subsequent crystalline phase transition more closely, a combination of SAXS/WAXS was employed. Figure 1b shows time-resolved SAXS curves for a reaction performed at $78{ }^{\circ} \mathrm{C}$. Upon the appearance of a crystalline phase, the correlation peak at $q \approx 2.4 \mathrm{~nm}^{-1}$ shows an instantaneous shift to $q \approx 1.8 \mathrm{~nm}^{-1}$ (at $t=20 \mathrm{~min}$; blue curves), followed by an additional shift to $q \approx 1.3 \mathrm{~nm}^{-1}$ after $40 \mathrm{~min}$ of reaction. Concurrently, the scattered intensity of the system increased by a factor of $\approx 1.4$. Extended reaction times showed a further shift of the correlation peak to even lower scattering angles, until a constant value of $q \approx 0.3 \mathrm{~nm}^{-1}$ was reached. At the end of the reaction, the absolute scattered intensity was a factor of $\approx 33$ higher than at 

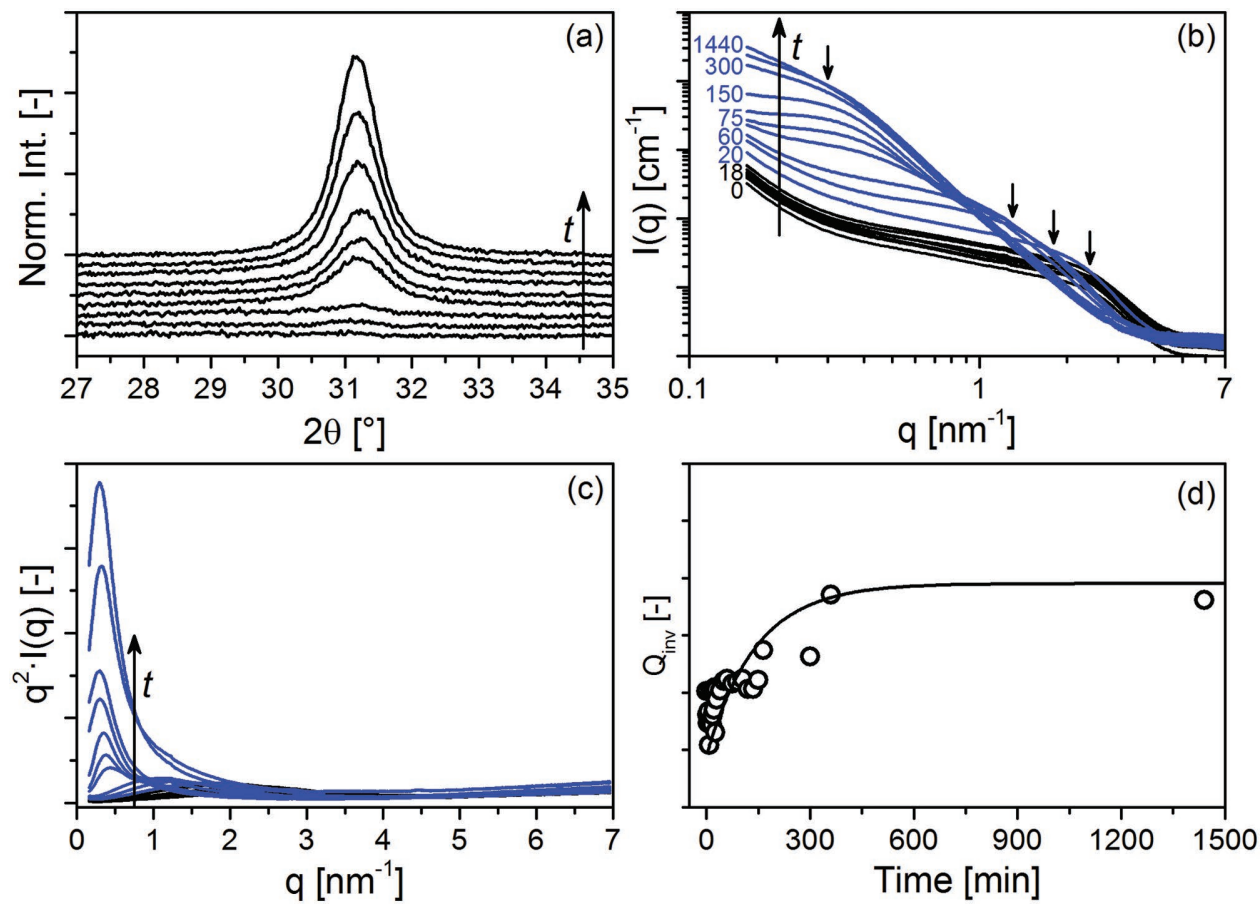

Figure 1. Time-resolved data obtained from a reaction performed at $78^{\circ} \mathrm{C}$ for stoichiometric precursor concentration of $0.2 \mathrm{~mol} \mathrm{dm}{ }^{-3}$. a) Evolution of (110) peak area from the XRD data. b) Shift of SAXS correlation peak to lower $q$ due to transformation from an amorphous (black line) to a crystalline (blue line) phase; correlation peak maxima are indicated by arrows. c) Kratky plot. d) Scattering invariant as a function of time.

the time of amorphous-to-crystalline transition. Moreover, a large linear regime is visible in the range $0.4<q<2.7 \mathrm{~nm}^{-1}$.

A similarly distinct difference between the amorphous (black curves) and crystalline phase was observed in the Kratky plot (Figure 1c). Here, the scattering angle $q$ is plotted versus $q^{2} \cdot I(q)$ to normalize the high contribution in scattered intensity of the largest particles (at low-q). Subsequent integration of the data in the Kratky plot yielded the for the low- $q$ regime (Guinier approximation; $0<q<0.16 \mathrm{~nm}^{-1}$ ) and high- $q$ regime (Porod law; $6.96<q<\infty \mathrm{nm}^{-1}$ ), scattering invariant (Figure 1d), which was corrected by extrapolation. The initial rapid increase of the scattering invariant signifies an increase in total scattering volume, imparted by the growth of crystallites. This corroborates well with the concurrent shift of the correlation peak from $q \approx 2.4$ to $0.3 \mathrm{~nm}^{-1}$ in Figure $1 \mathrm{~b}$. After $t \approx 450 \mathrm{~min}$, a plateau was reached, and no further changes in the scattering volume were observed, implying that further crystallite growth did not occur beyond this time. A closer look at Figure 1b shows quite different slopes in $I(q)$ for $q<0.2 \mathrm{~nm}^{-1}$. The steep increase in $I(q)$ during the first $60 \mathrm{~min}$ of the reaction suggests the existence of a large network or gel (with dimensions far greater than observable in the measured q-range) in which nucleation and growth of the crystallites occurred. In the time interval from 75 to $150 \mathrm{~min}$, however, this slope was absent, and only small changes in the position of the correlation peak were observed. This suggests that gel-like network ceased to exist and no further crystallite growth occurred beyond this time. The sharp rise in scattered intensity at low $q$ observed $>300 \mathrm{~min}$, may be interpreted as clustering of crystallites to aggregate sizes beyond the experimental q-range. However, the presence of a correlation peak at $q \approx 0.3 \mathrm{~nm}^{-1}$ suggests that crystallites did not grow further through coalescence, but rather through clustering of discrete crystallites into larger agglomerates. This agrees well with results from a previous study in which no growth beyond $10 \mathrm{~nm}$ sized crystallites was observed due to the presence of a dense capping layer on the crystallites' surface. ${ }^{[8 b]}$ In addition, the near-constant values for $Q_{\text {inv }}$ for reaction times $>300$ min proved that no further changes in the scattering volume, and thus growth through precipitation, occurred (Figure 1d).

\subsection{Nucleation and Growth}

To further investigate the effect of precursor concentration on the nucleation and growth mechanisms, particle size distributions (PSDs) were extracted from the SAXS curves (see Figure 2). From the obtained size distribution it is apparent that a great number of tiny crystallites $(1-3 \mathrm{~nm}$ ) were formed in the early stages of the reaction. These crystallite sizes coincide well with the sizes obtained from the corresponding position of the correlation peaks in Figure $1 \mathrm{~b}$ (for $1.3<q<2.4 \mathrm{~nm}^{-1}$ ). As the Ti- and Ba-precursor concentration was decreased from 0.2 to $0.075 \mathrm{~mol} \mathrm{dm}^{-3}$, we observe that the total number of smallest crystallites was reduced, while crystallization times increased from 20 to $120 \mathrm{~min}$, respectively. Due to a lack of a sufficient number of smallest crystallites, no crystallization time could be determined for the lowest concentration. We hypothesize that the small number of crystallites not only depends on the decreased precursor concentration, but also on the slower kinetics of hydrolysis and condensation due to the lower availability of water (which inadvertently is lowered due to lower 


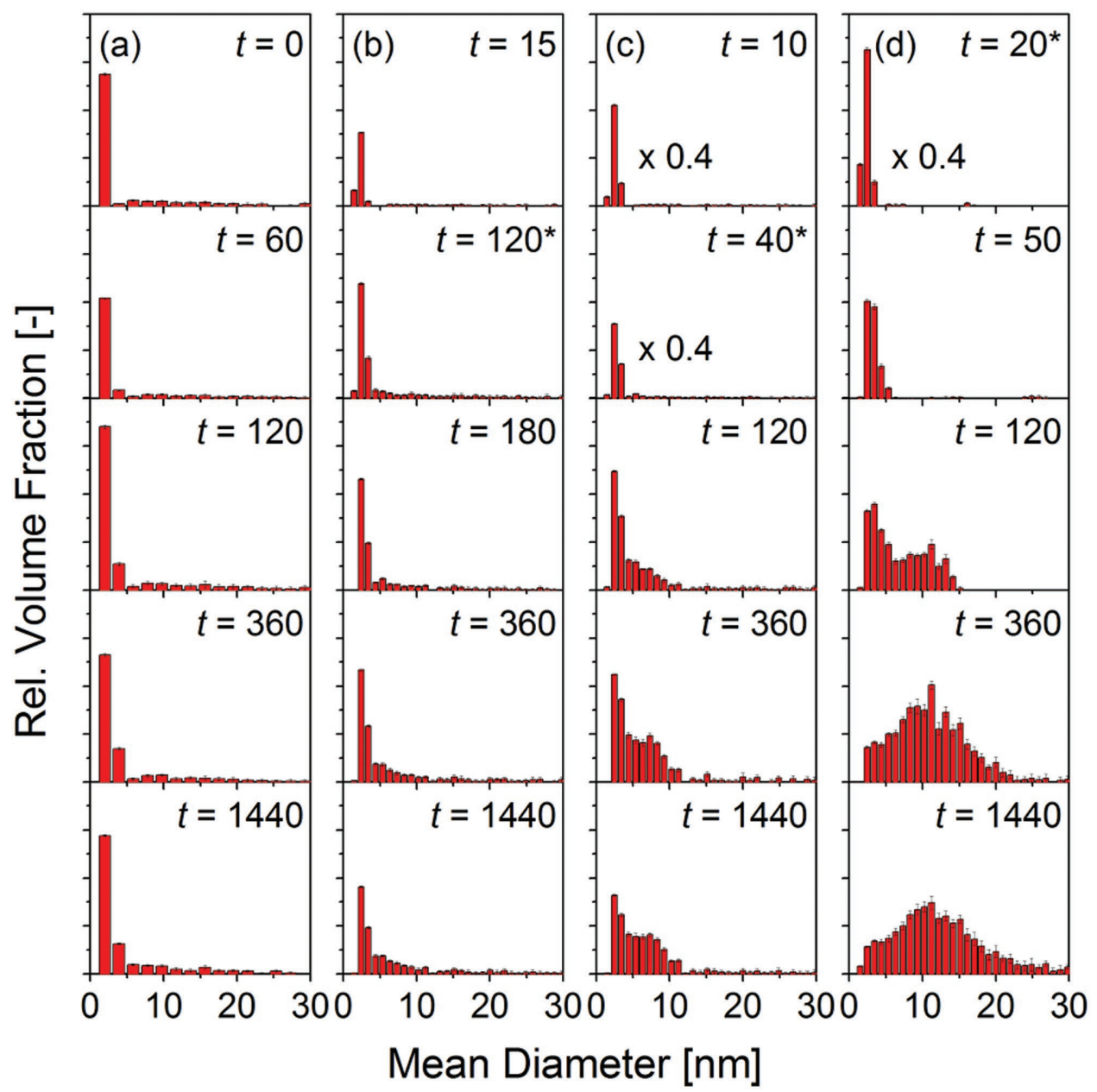

Figure 2. Particle size distributions obtained by fitting SAXS curves with the McSAS software, for experiments performed at $78^{\circ} \mathrm{C}$ and $\mathrm{Ti}\left(\mathrm{O}^{\prime} \mathrm{Pr}\right){ }_{4}$ concentrations of a) 0.05 , b) 0.075 , d) 0.1 , and d) $0.2 \mathrm{~mol} \mathrm{dm}^{-3}$. The plots where the time points (in min) are denoted with an asterisk correspond to the appearance of the crystalline phase in XRD and WAXS. The $y$-axes are all equally scaled.

starting concentrations of the Ba salt). The final crystallite sizes (mean value) extracted for $\mathrm{Ti}\left(\mathrm{O}^{I} \mathrm{Pr}\right)_{4}$ is $0.05,0.075,0.1$, and $0.2 \mathrm{~mol} \mathrm{dm}^{-3}$ were $4.0,5.6,6.4$, and $10.0 \mathrm{~nm}$, respectively.

High-resolution transmission electron microscopy (HR-TEM) was then used to visualize the size and morphology of the synthesized crystallites (Figure 3). After $24 \mathrm{~h}$ of reaction, only a handful of very small crystallites, embedded in an amorphous Ti-based matrix, were observed

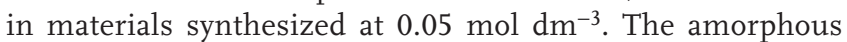
nature of the Ti-based matrix was confirmed with electron diffraction (see Figure 3a). At $0.075 \mathrm{~mol} \mathrm{dm}^{-3}$ more BTO crystallites were formed, however, they were still embedded in an amorphous matrix. Due to the increased amount of crystalline material, a polycrystalline diffraction pattern was obtained (see Figure 3b inset and Supporting Information for the corresponding diffraction planes). A further increase in concentration led to the disappearance of the amorphous network and subsequent increase in the volume fraction of crystalline particles (as observed with XRD, Figure 1a). The consumption of an initial hydrous Ti gel was also observed by MacLaren et al. after prolonged heating at $80{ }^{\circ} \mathrm{C}$ under hydrothermal conditions; ${ }^{[19]}$ albeit in their synthesis the $\mathrm{Ba}$ precursor was dissolved in water, and thus an enormous excess was available for the hydrolysis of Ti compared to our synthesis. Similarly, Viviani et al. observed the growth of spherical crystalline BTO particles $(<10 \mathrm{~nm})$ at the expense of larger amorphous particles $(\approx 100-200 \mathrm{~nm}),{ }^{[20]}$ whereby the crystalline phase was only found in the sub-10 nm-sized particles. These findings were used to infer that nanosized BTO crystals form initially within an amorphous matrix, and the subsequent coalescence into larger crystals occurs after the collapse of the gel. In our samples, the disappearance of the $\mathrm{Ti}$ gel at $[\mathrm{Ti}]>0.1 \mathrm{~mol} \mathrm{dm}^{-3}$ also suggests that crystallite growth occurred at the expense of the amorphous phase. Interestingly, the formation and crystallization of very small crystallites was induced after prolonged irradiation of the focused electron beam on a single spot (observed in sample $[\mathrm{Ti}]=0.05 \mathrm{~mol} \mathrm{dm}{ }^{-3}$; data not shown), suggesting that $\mathrm{Ba}^{2+}$ ions were present within the gel matrix. Given the porous nature of these condensed Ti networks, high concentrations of adsorbed $\mathrm{Ba}^{2+}$ ions may be expected on the gel's surface. ${ }^{[19,21]}$ Favorable conditions to crystallize may therefore also be dependent on the availability of sufficiently high $\mathrm{Ba}$ concentrations within the amorphous network. ${ }^{[22]}$ 


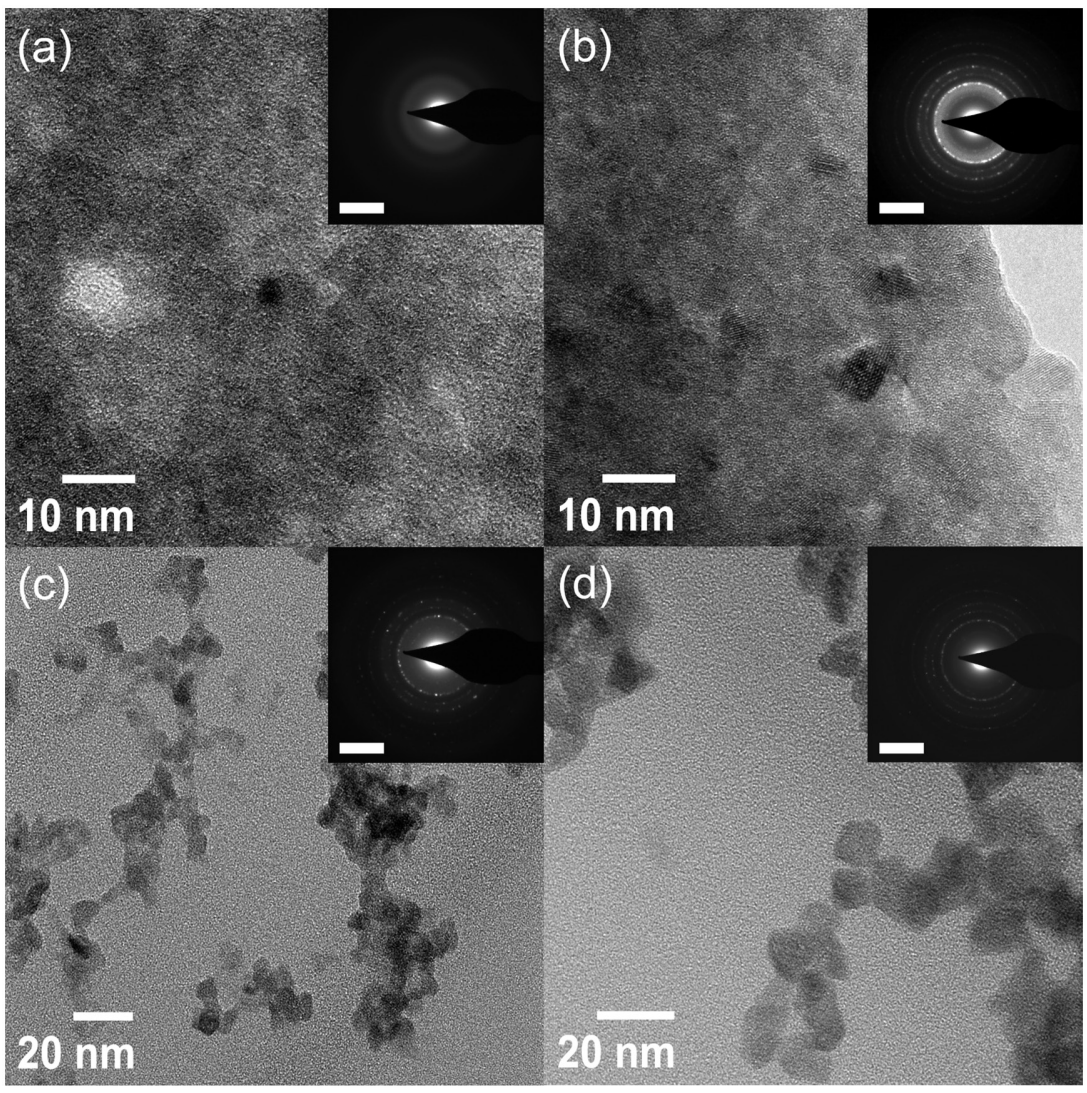

Figure 3. HR-TEM images of BTO synthesized at $78^{\circ} \mathrm{C}$ ( $24 \mathrm{~h}$ reaction time) using various concentrations: a) 0.05 , b) 0.075 , c) 0.1 , and d) $0.2 \mathrm{~mol} \mathrm{dm}^{-3}$. Insets are selected-area electron diffraction (SAED) patterns acquired from typical areas as shown in the images; all scale bars in the SAED pattern are $4 \mathrm{~nm}^{-1}$.

The scattering invariant provides access to several parameters that enable a better understanding of the nucleation and growth process of BTO in benzyl alcohol. The specific surface area, defined as the ratio between $\langle A\rangle$ and $V_{\mathrm{p}}$ provides a good measure for the appearance of small nuclei (large $\left.\langle A\rangle / V_{\mathrm{p}}\right)$ and their subsequent growth into larger clusters/crystallites (smaller $\langle A\rangle / V_{\mathrm{p}}$ ), while number density changes (with changing electron density contrast) of the emerging new phase(s) can be monitored via Equation (9). An overview of the derived surface-to-volume and number density changes, in relation to the average crystallite size and scattering length $\langle L\rangle$ are presented in Figure 4. The synthesis performed using the highest tested concentration $\left(0.2 \mathrm{~mol} \mathrm{dm}^{-3}\right.$; Figure $\left.4 \mathrm{~d}\right)$ shows the rapid emergence of a vast number of stable primary clusters, as witnessed by the fast increase in both $N \cdot(\Delta \rho)^{2}$ and $\langle A\rangle / V_{\mathrm{p}}$, while their subsequent growth is characterized by the rapid decay of both curves and the concurrent stark increase in $\langle L\rangle$. The peak for $N \cdot(\Delta \rho)^{2}$ at $\approx 65$ min coincides with the disappearance of the gel matrix and the sudden shift of the correlation peak from $q \approx 1.3$ to $0.3 \mathrm{~nm}^{-1}$ (see Figure $1 \mathrm{~b}$ ). The rapid decrease in the product $N \cdot(\Delta \rho)^{2}$, implies a strong reduction of the contribution of $N$, in which the scattering contrast between the newly evolved phase and the supernatant liquid has reached its maximum value.

Due to the fast growth rate, these larger crystallites are thus most likely formed through the aggregation and/or coalescence of smaller crystallites. Similarly, the fast decay observed for $\langle A\rangle / V_{\mathrm{p}}$ at $\approx 105$ min further explains the loss of surface-to-volume ratio due to the rapid crystallite growth. At the start of the coalescence, the surface area of as-formed aggregates is still high, due to the surface area contribution of the individual crystals. Over time, this contribution was reduced as a result of particle smoothening, leading to a decrease of $\langle A\rangle$. The fact that $\langle A\rangle / V_{\mathrm{p}}$ decayed $\approx 40$ min later than $N \cdot(\Delta \rho)^{2}$ may thus be explained by the time required to create smooth crystallites. A plateau was reached for both parameters after crystal growth stopped. For the lowest concentration $\left(0.05 \mathrm{~mol} \mathrm{dm}^{-3}\right.$; Figure 4a) no real nucleation burst of primary particles (amorphous or crystalline) was observed. The rather gradual change in specific surface area suggests the appearance and dissolution of small (unstable) clusters, until their precipitation onto larger (more stable) clusters. ${ }^{[23]}$ At these low concentrations the growth rate is probably limited by the low monomer diffusion rate, which mechanism is known to favor the formation of monodisperse distributions. ${ }^{[24]}$ No (real) growth beyond $4 \mathrm{~nm}$ sized (in diameter) crystallites was observed, corroborating well with the particle size distribution in Figure 2a. At intermediate concentrations $\left(0.075-0.1 \mathrm{~mol} \mathrm{dm}^{-3}\right.$; Figure $\left.4 \mathrm{~b}, \mathrm{c}\right)$ the absence of a peak in $\langle A\rangle / V_{\mathrm{p}}$ indicates that the burst nucleation is less pronounced due to the gradual growth of stable crystallites, while the absence of a peak in $N \cdot(\Delta \rho)^{2}$ suggests that further particle coalescence does not take place (i.e., with a constant number of particles. This corroborates well with the narrow particle size distributions observed for precursor concentrations $\leq 0.1 \mathrm{~mol} \mathrm{dm}^{-3}$, and a broader distribution for [Ti] $=0.2 \mathrm{~mol} \mathrm{dm}^{-3}$ (Figure 2).

\subsection{Varying Reaction Conditions}

\subsubsection{Temperature Dependence $\left([\mathrm{Ba}]=[\mathrm{Ti}]=0.2 \mathrm{~mol} \mathrm{dm}^{-3}\right)$}

Above, we have only discussed the effect of the precursor concentration on the formation of BTO nanocrystals at $78^{\circ} \mathrm{C}$. Since our previous study showed that the BTO crystallization process in benzyl alcohol was thermally activated, we have further investigated the effect of reaction temperature (i.e., 45 and $150^{\circ} \mathrm{C}$ ) on the nucleation and growth processes. ${ }^{\left[{ }^{[b]}\right]}$ The results (Figure $4 \mathrm{e}, \mathrm{f}$ ) allowed us to derive a crystallization activation energy of $E_{\mathrm{A}}=$ $65.2 \mathrm{~kJ} \mathrm{~mol}^{-1}$ over the temperature range between 45 and $150^{\circ} \mathrm{C}$. This is comparable to $E_{\mathrm{A}}=55.1 \mathrm{~kJ} \mathrm{~mol}^{-1}\left(150-200^{\circ} \mathrm{C}\right)$ found for the crystallization of BTO from a hydrothermal reaction using a hydrous amorphous titania precursor. ${ }^{[25]}$ Furthermore, we showed previously that the water release from the Ba hydroxide precursor follows a zeroth order reaction rate (i.e., rate independent of the precursor concentration), whereby similar water 

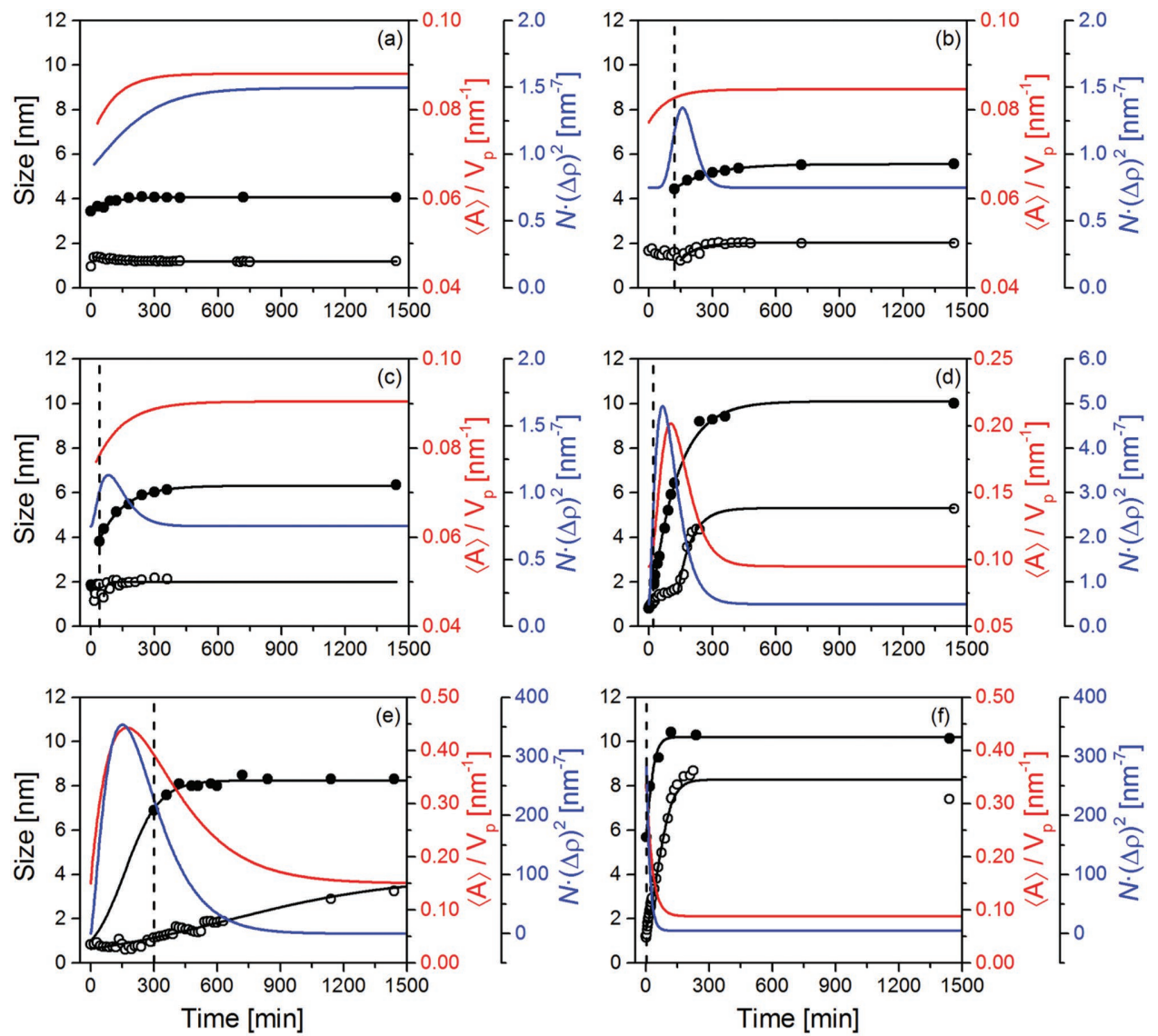

Figure 4. Schematic overview of the mean crystallite size (black circles), scattering length $\langle L\rangle$ (open circles), specific surface area $\langle A\rangle / V_{p}$ (in $n m^{-1}$; red line), and the product of particle number and scattering density $N \cdot(\Delta \rho)^{2}$ (in $\mathrm{nm}^{-7}$; blue line) for reactions performed with [Ba]:[Ti] $=1$ and concentration a) 0.05 , b) 0.075 , c) 0.1 , and d) $0.2 \mathrm{~mol} \mathrm{dm}^{-3}$. e,f) Reactions performed with [Ba]:[Ti] $=1$ and $0.2 \mathrm{~mol} \mathrm{dm}^{-3}$ at 45 and $150{ }^{\circ} \mathrm{C}$, respectively. The dashed vertical lines represent the times of crystallization determined by XRD, and serve only as a guide to the eye. For clarity, the red/blue curves are presented as average curves (see Figure S2 for example).

amounts were released at varying reaction temperatures, for a given concentration. ${ }^{[8 \mathrm{~b}]}$ In other words, the dehydration is faster at higher temperatures, thus affecting both nucleation and crystallization rates. More importantly, the solubility of the barium hydroxide in its released hydrated water is strongly temperature-dependent (see Figure S3, Supporting Information). ${ }^{[26]}$ Consequently, the limited solubility at reaction temperatures $<60{ }^{\circ} \mathrm{C}$ causes a temporal saturation of $\mathrm{Ba}^{2+}$ ions due to the slower release of hydrated water, which is independent of the initial barium hydroxide octahydrate concentration. This effect is negated at reaction temperatures above the Ba salt's melting point due to the significantly higher solubility. On the other hand, the released hydrated water is also prerequisite to hydrolyze the Ti precursor, which is of great importance for the selforganization toward crystallization. Its high reactivity, however, ensures a rapid release of the used water for hydrolysis upon alkoxilation (i.e., Ti-O-Ti network formation). The hydrolysis of titanium (IV) isoproxide thus always outcompetes the dissolution of $\mathrm{Ba}^{2+}$ ions, and is therefore not the rate-limiting step. Our data confirmed that, at $45^{\circ} \mathrm{C}$ (Figure 4e), nucleation and growth took place over a much longer period of time due to the and limited availability of dissolved $\mathrm{Ba}^{2+}$ ions caused by the slower release of water, whereas at $150{ }^{\circ} \mathrm{C}$ (Figure $4 \mathrm{f}$ ), the reaction proceeded very rapidly. Intermediate trends observed for the reaction performed at $78^{\circ} \mathrm{C}$ (Figure $4 \mathrm{~d}$ ) indicate that the rates of hydrolysis and subsequent crystallization and growth processes are indeed determined by the release rate of $\mathrm{Ba}$ hydration water.

\subsubsection{Influence of Water $\left([\mathrm{Ba}]=[\mathrm{Ti}]=0.05-0.2 \mathrm{~mol} \mathrm{dm}^{-3}\right)$}

The concentration of water present in the system has a profound influence on the manner in which the reaction proceeds. An excess of water may lead to uncontrolled precipitation and growth of crystallites, ${ }^{[7,27]}$ while a lack of water may result in no reaction at all. The barium hydroxide octahydrate precursor was used for the controlled release of seven moles of water per mole of Ba during heating. ${ }^{[8 b]}$ Thus, the reduction of the overall $\mathrm{Ti}$ (and $\mathrm{Ba}$ ) precursor concentration from 0.2 to $0.05 \mathrm{~mol} \mathrm{dm}^{-3}$ effectively reduced the overall water concentration in the system from 2.46 to 0.63 vol\%, respectively, while keeping $h=\left[\mathrm{H}_{2} \mathrm{O}\right]:[\mathrm{Ti}]=7$. The zeroth order dehydration reaction of the $\mathrm{Ba}$ precursor ensured equal water release rates for all investigated concentrations, although the total water concentration available for hydrolysis was four times higher for $0.2 \mathrm{~mol} \mathrm{dm}^{-3}$ than for $0.05 \mathrm{~mol} \mathrm{dm}^{-3}$. 


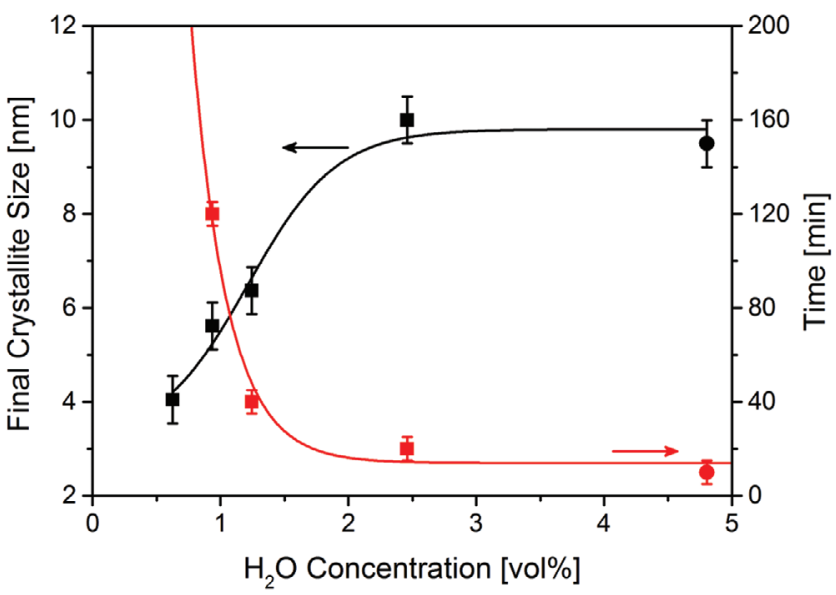

Figure 5. Influence of the overall volume percentage of water on the crystallization time and final mean crystallite size (determined by PSD from SAXS curves). Varying precursor concentrations $\left([\mathrm{Ti}]=0.05-0.2 \mathrm{~mol} \mathrm{dm}^{-3}\right.$ ) are denoted by squares, whereas samples prepared with a precursor stoichiometry $\mathrm{Ba}: \mathrm{Ti}=2: 1 \quad\left(h=14 ;[\mathrm{Ti}]=0.2 \mathrm{~mol} \mathrm{dm}^{-3}\right)$ are denoted by circles. Note: the crystallization time of [Ti] $=0.05 \mathrm{~mol} \mathrm{dm}^{-3}$ could not be determined.

Figure 5 shows that an increased $\mathrm{Ti}$ concentration led to reduced crystallization times and increased mean crystallite sizes (red and black squares, respectively). It is also evident that not the $\left[\mathrm{H}_{2} \mathrm{O}\right]:[\mathrm{Ti}]$ ratio, but rather the total water concentration determines the rate of crystallization (see also Table 1 for an overview of all reaction conditions and crystallization times). To investigate this issue further, two additional experiments were performed: (1) the water content of a reaction performed with [Ti] $=0.1 \mathrm{~mol} \mathrm{dm}^{-3}$ was carefully adjusted from 1.25 to $2.46 \mathrm{vol} \%$ ( $h=7$ to 14 , respectively); (2) a reaction was performed with $[\mathrm{Ti}]=0.4 \mathrm{~mol} \mathrm{dm}^{-3}\left(4.80 \mathrm{vol} \% \mathrm{H}_{2} \mathrm{O} ; h=7\right)$.Testing the influence of the increase of $h$ by a factor 2 , and the influence of the total water concentration on the crystallization time, respectively, revealed that a crystalline phase started to appear after $\approx 20 \mathrm{~min}$, and independent of the precursor concentration and $h$, comparable times for crystallization were found as for [Ti] = $0.2 \mathrm{~mol} \mathrm{dm}^{-3}$. Above a critical water concentration of $2.46 \mathrm{vol} \%$ (i.e., complete solubility of available $\mathrm{Ba}^{2+}$ ions), the crystallization proceeded seemingly via a zeroth order reaction rate, corresponding to the dehydration rate of barium hydroxide octahydrate. Moreover, crystallization was not affected by the shorter distances over which diffusion of reactive species has to occur for $[\mathrm{Ti}]=0.4 \mathrm{~mol} \mathrm{dm}^{-3}$ compared to $0.2 \mathrm{~mol} \mathrm{dm}^{-3}$. This implies that the gel formation rate (through subsequent hydrolysis and condensation reactions) is rate-limiting, rather than the flux or diffusion of $\mathrm{Ba}^{2+}$ ions. For [Ti] $=0.1 \mathrm{~mol} \mathrm{dm}^{-3}$, below the water threshold of $2.46 \mathrm{vol} \%$, the amorphous network formation was impeded by the lower supply of water. Although the hydrolysis and condensation reactions are expected to be very rapid in the presence of water, ${ }^{[7]}$ the diffusion of water molecules towards reactive Ti species was limiting the reaction rate. The crystalline phase was formed after $40 \mathrm{~min}$; exactly twice as long as for $[\mathrm{Ti}]=0.2 \mathrm{~mol} \mathrm{dm}^{-3}$. At $[\mathrm{Ti}]<0.1 \mathrm{~mol} \mathrm{dm}^{-3}$, the time of crystal formation was not linearly related to the precursor concentration. Hence, the crystallization reaction was presumably limited by the diffusion rate of water molecules, and thus the competition between the amorphous titania network formation and the solubilization of $\mathrm{Ba}^{2+}$ ions.

\subsubsection{Influence Ba:Ti Stoichiometry ([Ti] $=0.2 \mathrm{~mol} \mathrm{dm}^{-3}$ )}

An increased Ba:Ti ratio of 2:1 (4.80 vol\% $\left.\mathrm{H}_{2} \mathrm{O} ; h=14\right)$ had no significant effect on the final crystallite size (Figure 5; black circle), but it reduced the crystallization time from 20 to $10 \mathrm{~min}$ (red circle), respectively. Although an excess of Ba was present, only an equimolar amount participates in the BTO formation reaction. Since the water concentration is sufficiently high, the reduction in crystallization time was thus linked to the decrease of the diffusive path length of $\mathrm{Ba}^{2+}$ ions. In later stages of the reaction, the excess of Ba resulted in the formation of secondary phases, such as $\mathrm{BaCO}_{3}$. The more water lean conditions in reactions performed with $\mathrm{Ba}: \mathrm{Ti}=0.5: 1\left(1.25\right.$ vol\% $\left.\mathrm{H}_{2} \mathrm{O} ; h=3.5\right)$ led to a crystallization time $>720 \mathrm{~min}$ (Table 1). Although the water concentration was equal to that in a stoichiometric reaction performed at $[\mathrm{Ti}]=0.1 \mathrm{~mol} \mathrm{dm}^{-3}$, the crystallization was significantly slower. The crystallization rate is determined from a correlation between the water concentration, $h$, the precursor stoichiometry and flux of water, the rate of gel formation, and the solubility/flux of $\mathrm{Ba}^{2+}$ ions, respectively. To clarify this relationship, the water concentration in reaction mixtures with [Ti]

Table 1. Overview of the influence of precursor concentration, reactant stoichiometry, and added water on the crystallization time.

\begin{tabular}{|c|c|c|c|c|c|}
\hline Ti Concentration $\left[\mathrm{mol} \mathrm{dm}^{-3}\right]$ & {$[\mathrm{Ba}]:[\mathrm{Ti}][-]$} & $\mathrm{H}_{2} \mathrm{O}$ added & $h=\left[\mathrm{H}_{2} \mathrm{O}\right]:[\mathrm{Ti}][-]$ & $\mathrm{H}_{2} \mathrm{O}$ Concentration [\%] & Crystallization time [min] \\
\hline 0.05 & 1 & No & 7 & 0.63 & n.d. \\
\hline 0.075 & 1 & No & 7 & 0.94 & 120 \\
\hline 0.1 & 1 & No & 7 & 1.25 & 40 \\
\hline 0.2 & 1 & No & 7 & 2.46 & 20 \\
\hline 0.1 & 1 & Yes & 14 & 2.46 & 22 \\
\hline 0.4 & 1 & No & 7 & 4.80 & 20 \\
\hline 0.2 & 2 & No & 14 & 4.80 & 10 \\
\hline 0.2 & 0.5 & No & 3.5 & 1.25 & $>720$ \\
\hline 0.2 & 0.5 & Yes & 7 & 2.46 & 40 \\
\hline 0.2 & 0.375 & Yes & 7 & 2.46 & 123 \\
\hline
\end{tabular}


$=0.2 \mathrm{~mol} \mathrm{dm}^{-3}$, two sub-stoichiometric Ba:Ti ratios, namely 0.5:1 (1.25 vol\% $\left.\mathrm{H}_{2} \mathrm{O} ; h=3.5\right)$ and $0.375: 1\left(0.94\right.$ vol\% $\mathrm{H}_{2} \mathrm{O} ; h=$ 2.6), was carefully adjusted to 2.46 vol\% (i.e., the water amount normally present for $\mathrm{Ba}: \mathrm{Ti}=1: 1 ; h=7)$. Effectively, both reactions should yield an equivalent amount of BTO as performed under stoichiometric conditions using [Ti] $=0.1$ and 0.075 mol dm${ }^{-3}$, respectively. Our hypothesis was confirmed by the near-identical reaction rates that were found for the wateradjusted reactions compared to the latter reactions performed under stoichiometric conditions (see Table 1). Thus, provided that sufficient water is supplied for hydrolysis and gel formation, the crystallization times are determined predominantly by the lower solubility, flux of $\mathrm{Ba}^{2+}$ ions, and the longer diffusion length towards the $\mathrm{Ti}$ atoms.

\subsection{Rate-Limiting Regimes}

To summarize the abovementioned results, the dependencies of the investigated reactions conditions on the rate-limiting regimes for crystal formation are presented in Figure 6. Three regimes were identified in which: (1) the gel formation (grey area), (2) the flux water and solubility and flux of $\mathrm{Ba}^{2+}$ ions (blue area), and (3) a combination of both (red area) are the rate-limiting factors. For reactions performed under stoichiometric precursor ratios, the crystallization rate is determined
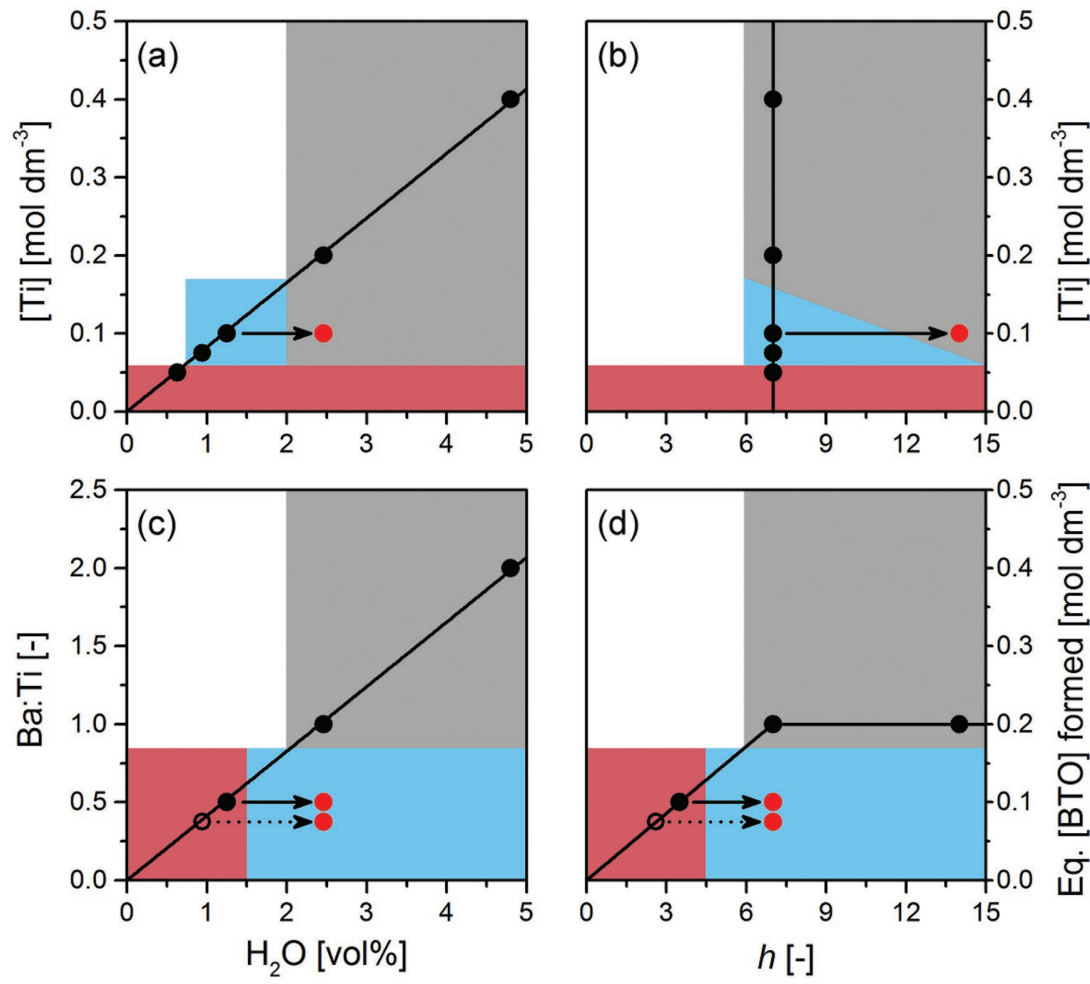

Figure 6. Overview of rate-limiting regimes for reactions performed under a,b) stoichiometric, and c,d) nonstoichiometric precursor conditions. The grey, blue, and red areas represent the regimes in which the gel formation rate, water concentration, and a combination of both are the rate-limiting conditions, respectively. The black circles represent reactions carried out without further adjustments. The open and red circles indicate reactions that were not carried out and reactions performed with adjusted water concentrations, respectively. by the amount of water and the rate at which it is released from the barium hydroxide octahydrate. For $[\mathrm{Ti}] \geq 0.2 \mathrm{~mol} \mathrm{dm}^{-3}$ sufficient water is provided by the $\mathrm{Ba}$ precursor to solubilize all $\mathrm{Ba}^{2+}$ ions, and crystal formation is limited by the dehydration of the Ba precursor, and thus the rate of gel formation. At lower concentrations, the distance between water molecules to the reactive $\mathrm{Ti}$ atoms, and thus the diffusion of water, rather than the formation of an amorphous network is rate-limiting. For reactions performed with nonstoichiometric precursor ratios, the rate of crystallization is not only determined by the absolute water concentration, but also by $h$. The hydrolysis and condensation reactions are severely impeded at $h<7$. In addition, variations in the Ba:Ti ratio change the diffusion length of $\mathrm{Ba}^{2+}$, and thus also influence the rate at which crystals are formed.

\section{Conclusions}

In complex chemical syntheses, such as coprecipitation reactions, nucleation, growth, and coarsening processes often occur concomitantly, whereby the individual processes are frequently obscured. We have discerned successfully the effect of reaction conditions on the nucleation, crystallization, and growth phenomena on nanocrystalline BTO formation by employing the scattering invariant. This form-free and model independent approach, obtained from combined timeresolved SAXS/WAXS allowed us to discern the nucleation, crystallization, and growth phenomena during the synthesis. The effect of precursor concentration, reactant stoichiometry, and temperature on the amorphousto-crystalline transformation was described in detail, and three rate-limiting regimes were established. Sufficiently high water concentrations during the reaction enabled a quick succession of the nucleation, crystallization, and growth events. Here, the rate of BTO nanocrystal formation was only limited by the dehydration rate of the Ba precursor, and thus by the formation of an amorphous gel network. Conversely, water lean conditions resulted in impeded gel formation and only a gradual nucleation, growth, and crystallization process was observed. For reaction performed under nonstoichiometric precursor ratios, not only the total water concentration, but also $h$ determined the reaction rate. The latter suggested that the changes in the diffusion length between $\mathrm{Ba}^{2+}$ ions and the reactive $\mathrm{Ti}$ atoms affected the kinetics. Lastly, since the scattering invariant approach measures the total scattered intensity, and does not require prior knowledge of the reaction mechanism (i.e., model independent), it can be extended to other synthesis methods and material's systems. A better understanding of the effect of modified reaction parameters on the synthesis of 
nanocrystals enable to set up elementary boundary conditions for improved reaction control.

\section{Experimental Section}

Chemicals and Materials: Titanium (IV) isopropoxide $\left(\mathrm{Ti}\left(\mathrm{O}^{\prime} \mathrm{Pr}\right)_{4}\right)$, $99.999 \%)$, barium hydroxide octahydrate $\left(\mathrm{Ba}(\mathrm{OH})_{2} \cdot 8 \mathrm{H}_{2} \mathrm{O}, 98.0 \%\right)$, and 2-propanol (99.5\%) were purchased from Sigma-Aldrich. Benzyl alcohol $(99.0 \%)$ was acquired from Acros. All chemicals were used as-received from the suppliers without any further purification. Both titanium (IV) isopropoxide and benzyl alcohol were stored and handled in a water-free environment $\left(<0.1 \mathrm{ppm} \mathrm{H}_{2} \mathrm{O}\right)$.

Formation of Crystalline Barium Titanate: Stoichiometric amounts (i.e., $[\mathrm{Ti}]=[\mathrm{Ba}]$ ) of barium hydroxide octahydrate were added to $0.05-0.2 \mathrm{~mol} \mathrm{dm}^{-3}$ solutions of titanium (IV) isopropoxide in benzyl alcohol. While stirring, the reaction mixture was heated to $78^{\circ} \mathrm{C}$ at a rate of $5{ }^{\circ} \mathrm{C} \mathrm{min}^{-1}$. At various time intervals, aliquots were taken from the reaction vessel, thermally quenched to stop the reaction, and subsequently stored at $-18{ }^{\circ} \mathrm{C}$. The samples used for XRD analysis were centrifuged using a Sigma 1-14 centrifuge at $14800 \mathrm{rpm}$ for $15 \mathrm{~min}$. The supernatant benzyl alcohol phase was removed, the precipitated BTO rinsed with 2-propanol, dried in air, and subsequently measured with XRD. More information on the synthesis can be found elsewhere. ${ }^{[8]}$ The effect of various experimental conditions on the synthesis reaction was investigated at $78^{\circ} \mathrm{C}$ by changing the $\left[\mathrm{Ti}\left(\mathrm{O}^{i} \mathrm{Pr}\right)_{4}\right]$ precursor concentrations $\left(0.05,0.075,0.1\right.$, and $\left.0.2 \mathrm{~mol} \mathrm{dm}^{-3}\right)$, the Ba:Ti ratios $(0.5: 1,1: 1$, and 2:1), and varying water content $\left(\left[\mathrm{H}_{2} \mathrm{O}\right] /[\mathrm{Ti}]=h=3.5,7\right.$, and 14$)$. To test the effect of temperature, experiments at $0.2 \mathrm{~mol} \mathrm{dm}^{-3}$ were also carried out at 45 and $150^{\circ} \mathrm{C}$. In addition, the effect of prehydrolysis (i.e., water was added to the Ti precursor solution prior to $\mathrm{Ba}(\mathrm{OH})_{2} \cdot 8 \mathrm{H}_{2} \mathrm{O}$ addition) with an equivalent of 14 moles $\mathrm{H}_{2} \mathrm{O}$ per Ti was also investigated.

Time-Resolved X-Ray Diffraction: The synthesized samples were characterized with powder XRD ( $\mathrm{Cu} \mathrm{K} \alpha$ irradiation) to confirm the formation of the crystalline $\mathrm{BaTiO}_{3}$ phase using an X'Pert Powder Pro (PANalytical, Almelo, The Netherlands) with a 1D PIXcel detector. Scans from $2 \theta=27^{\circ}-35^{\circ}$ of the (110) peak were measured with step sizes of $0.026^{\circ}$ and $600 \mathrm{~s}$ per step. The patterns were further analyzed using the $X$ 'Pert Highscore Plus software package (version 3.0e).

SAXS: SAXS experiments were performed on the Dutch-Belgian beam line (BM-26B) of the European Synchrotron Radiation Facility (ESRF) in Grenoble, France. ${ }^{[28]}$ The X-ray beam energy (16 keV; $\lambda=0.0776 \mathrm{~nm}$ ) was positioned on the corner of a 2D Pilatus $1 \mathrm{M}$ detector to maximize the range of scattering angles. Ag behenate was used to calibrate the absolute scattering vector q-range $\left(\mathrm{nm}^{-1}\right)$ in the experiments. The detector was placed at a distance of $1.5 \mathrm{~m}$ from the sample, which allowed to record the effective scattering vector magnitude in the range of $0.16<q<6.96 \mathrm{~nm}^{-1}$. Samples were measured in sealed glass capillaries $(\varnothing=1.5 \mathrm{~mm}$; glass no. 50 ; Hilgenberg, Malsfeld, Germany) at room temperature. The absolute scattered intensity for all samples was calibrated with distilled water. ${ }^{[29]}$ The absolute scattered intensity was not determined for experiments performed at 45 and $150^{\circ} \mathrm{C}$, and the absolute values for $N \cdot(\Delta \rho)^{2},\langle A\rangle / V_{p}$, and $\langle L\rangle$ may deviate slightly. The data were only used to demonstrate the observed trends. For all measurements, the scattering of an empty capillary was subtracted as a background signal.

$P S D$ : Form-free particle size distributions were obtained from scattering curves using the Monte Carlo based software package McSAS. [14] All scattering curves were fitted in a range of 0.16 $<q<6.96 \mathrm{~nm}^{-1}$ (see Supporting Information for exemplary fits).

HR-TEM: Samples were investigated by HR-TEM (Philips CM300ST-FEG) with an acceleration voltage of $285 \mathrm{keV}$ using a Gatan Ultrascan 1000 CCD camera and further analyzed using the Image) processing software package (version 1.47q). ${ }^{[30]}$ SAED images were acquired with $5-10 \mathrm{~s}$ exposure time (10 frames) from typical areas of $\approx 0.9 \times 0.9 \mu \mathrm{m}^{2}$ and $0.2 \times 0.2 \mu \mathrm{m}^{2}$ for [Ti] $\leq 0.075 \mathrm{~mol} \mathrm{dm}^{-3}$ and $[\mathrm{Ti}] \geq 0.1 \mathrm{~mol} \mathrm{dm}^{-3}$, respectively.

\section{Supporting Information}

Supporting Information is available from the Wiley Online Library or from the author.

\section{Acknowledgements}

This work was financially supported by the Advanced Dutch Energy Materials Program (ADEM). the authors thank the Netherlands Organisation for Scientific Research (NWO) for granting us beam time at ESRF in Grenoble, France, and also acknowledge the assistance of the DUBBLE beam line (BM-26B) specialists, in particular Dr. W. Bras, Dr. G. Portale, and Dr. D. Hermida Merino. The expertise and help of Dr. E.G. Keim (MESA+) with HR-TEM is kindly acknowledged.

\section{Conflict of Interest}

The authors declare no conflict of interest.

\section{Keywords}

model independent analysis, perovskites, reaction kinetics, small angle $\mathrm{X}$-ray scattering, sol-gel

Received: May 25, 2018

Revised: August 19, 2018

Published online:

[1] J. Park, J. Joo, G. K. Soon, Y. Jang, T. Hyeon, Angew. Chem., Int. Ed. 2007, 46, 4630.

[2] C. B. Murray, D. J. Norris, M. G. Bawendi, J. Am. Chem. Soc. 1993, $115,8706$.

[3] a) D. Aldakov, A. Lefrançois, P. Reiss, J. Mater. Chem. C 2013, 1, 3756; b) S. V. Kershaw, A. S. Susha, A. L. Rogach, Chem. Soc. Rev. 2013, 42, 3033.

[4] L. Protesescu, S. Yakunin, M. I. Bodnarchuk, F. Krieg, R. Caputo, C. H. Hendon, R. X. Yang, A. Walsh, M. V. Kovalenko, Nano Lett. 2015, 15, 3692.

[5] a) R. Deshmukh, M. Niederberger, Chem. - Eur. J. 2017, 23, 8542; b) R. Deshmukh, M. Niederberger, in The Sol-Gel Handbook, (Eds: M. Zayat, D. Levy), Vol. 1, Wiley-VCH, Weinheim 2015, pp. 29-70.

[6] M. Niederberger, Acc. Chem. Res. 2007, 40, 793.

[7] C. J. Brinker, G. W. Scherer, Sol-Gel Science: The Physics and Chemistry of Sol-Gel Processing, Academic Press, San Diego, CA 1990.

[8] a) T. M. Stawski, S. A. Veldhuis, O. F. Göbel, J. E. ten Elshof, D. H. A. Blank, J. Am. Ceram. Soc. 2010, 93, 3443; b) S. A. Veldhuis, W. J. C. Vijselaar, T. M. Stawski, J. E. ten Elshof, Inorg. Chem. 2014, 53, 13188.

[9] B. L. Cushing, V. L. Kolesnichenko, C. J. O'Connor, Chem. Rev. 2004, 104, 3893.

[10] a) X. Chen, J. Schröder, S. Hauschild, S. Rosenfeldt, M. Dulle, S. Förster, Langmuir 2015, 31, 11678; b) S. Saha, S. Springer, M. E. Schweinefuß, D. Pontoni, M. Wiebcke, K. Huber, Cryst. Growth Des. 2016, 16, 2002

[11] a) A. Guinier, G. Fournet, C. B. Walker, K. L. Yudowitch, Small-Angle Scattering of X-Rays, Vol. 14, Wiley, New York 1955; b) J. S. Pedersen, Adv. Colloid Interface Sci. 1997, 70, 171. 
[12] J. Teixeira, J. Appl. Crystallogr. 1988, 21, 781.

[13] a) H. G. Krauthäuser, W. Lennartz, G. Nimtz, J. Appl. Crystallogr. 1996, 29, 7; b) S. Martelli, P. E. Di Nunzio, Part. Part. Syst. Charact. 2002, 19, 247.

[14] B. R. Pauw, J. S. Pedersen, S. Tardif, M. Takata, B. B. Iversen, J. Appl. Crystallogr. 2013, 46, 365.

[15] G. Porod, in Small Angle X-Ray Scattering (Eds: O. Glatter, O. Kratky), Academic Press, London 1982, pp. 17-51.

[16] J. Moller, R. Kranold, J. Schmelzer, U. Lembke, J. Appl. Crystallogr. 1995, 28, 553.

[17] P.-z. Wong, A. J. Bray, J. Appl. Crystallogr. 1988, 21, 786.

[18] R. Besselink, T. M. Stawski, A. E. S. V. Driessche, L. G. Benning, J. Chem. Phys. 2016, 145, 211908.

[19] I. MacLaren, C. B. Ponton, J. Eur. Ceram. Soc. 2000, 20, 1267.

[20] M. Viviani, M. T. Buscaglia, A. Testino, V. Buscaglia, P. Bowen, P. Nanni, J. Eur. Ceram. Soc. 2003, 23, 1383.

[21] D. Hennings, G. Rosenstein, H. Schreinemacher, J. Eur. Ceram. Soc. 1991, 8, 107
[22] J. A. Kerchner, J. Moon, R. E. Chodelka, A. A. Morrone, J. H. Adair, ACS Symp. Ser. 1998, 681, 106.

[23] R. Viswanatha, D. D. Sarma, in Nanomaterials Chemistry, (Eds: C. N. R. Rao, A. Müller, A. K. Cheetham), Wiley-VCH, Weinheim 2007, pp. 139-170.

[24] G. Cao, Nanostructures and Nanomaterials: Synthesis, Properties \& Applications, 1st ed., Imperial College Press, London, UK 2004.

[25] R. I. Walton, F. Millange, R. I. Smith, T. C. Hansen, D. O'Hare, J. Am. Chem. Soc. 2001, 123, 12547.

[26] H. L. Clever, I. Lambert, Alkaline Earth Hydroxides in Water and Aqueous Solutions, Pergamon, Amsterdam 1992, pp. 284-342.

[27] T. Sugimoto, X. Zhou, A. Muramatsu, J. Colloid Interface Sci. 2002, 252, 339.

[28] W. Bras, I. P. Dolbnya, D. Detollenaere, R. Van Tol, M. Malfois, G. N. Greaves, A. J. Ryan, E. Heeley, J. Appl. Crystallogr. 2003, 36, 791.

[29] L. Fan, M. Degen, S. Bendle, N. Grupido, J. Ilavsky, J. Phys.: Conf. Ser. 2010, 247, 012005.

[30] C. A. Schneider, W. S. Rasband, K. W. Eliceiri, Nat. Methods 2012, 9, 671. 